mean age, 61 years) were observed during the last 4 years. Coronary artery disease was diagnosed by clinical and ECG data and/or history of myocardial infarction. SMI was detected by ambulatory 24-hour ECG monitoring and VD were compared during periods with and without SMI. VD were classified in Lown's grades. The table summarizes the results.

\begin{tabular}{lcccc}
\hline \multirow{2}{*}{$\begin{array}{l}\text { Ambulatory } \\
\text { ECG Periods }\end{array}$} & \multicolumn{4}{c}{ Lown's Grade } \\
\cline { 2 - 5 } & I/II & III & IV A & IV B \\
\hline With SMI & 24 & 6 & 5 & 3 \\
& $(34 \%)$ & $(8 \%)$ & $(7 \%)$ & $(4 \%)$ \\
Without ischemia & 30 & 10 & 1 & 1 \\
& $(42 \%)$ & $(14 \%)$ & $(1 \%)$ & $(1 \%)$ \\
\hline
\end{tabular}

Conclusion. High-grade VD were more prevalent during periods of SMI, compared with periods without myocardial ischemia.

\title{
Ventricular Dysrhythmias During Silent Myocardial Ischemia
}

F. Pinto, M. Fiuza, E. Dias, J. Correia, P. Pedro, F. Ribeiro, F. de Pádua, University Center of Cardiology, Lisbon, Portugal

To analyze the prevalence of ventricular dysrhythmias (VD) during periods of silent myocardial ischemia (SMI), 71 consecutive patients with SMI (49 men, 22 women; 